\title{
Influence of the particle shape on the impact force of lahar on an obstacle
}

\author{
Rime Chehade ${ }^{1 *}$, Bastien Chevalier ${ }^{1}$, Fabian Dedecker $^{2}$, and Pierre Breul ${ }^{1}$ \\ ${ }^{1}$ Université Clermont Auvergne, CNRS, Clermont Auvergne INP, Institut Pascal, F-63000 Clermont-Ferrand, France \\ ${ }^{2}$ Itasca consultants SAS, 29 Avenue Joannes Masset, F-69009 Lyon, France
}

\begin{abstract}
Lahars represent natural phenomena that can generate severe damage in densely populated urban areas. The evaluation of pressures generated by these mass flows on constructions (buildings, infrastructure...) is crucial for civil protection and assessment of physical vulnerability. The existing tools to model the spread of flows at large scale in densely populated urban areas remain inaccurate in the estimation of mechanical efforts. A discrete numerical model is developed for evaluating debris flow (DF) impact pressures at the local scale of one structure. The large-sized solid particles are modelled explicitly using Distinct Element Method (DEM) and the fine-grained solid particles are integrated in a fluid phase which generates two effects on the movement of particles, i.e. buoyancy and drag. Fluid velocity field and the fluid free surface are obtained from Computational Fluid Dynamics (CFD) code then imported in the DEM simulation in a one way coupling scheme. In this paper, the influence of particle shape on the impact forces generated on the obstacle is investigated: spherical particles and polygonal rigid blocks (r-blocks) are considered. The shape of the particle influences the contact surface and therefore the impact pressure. With an angular shape and several facets like r-blocks, the impact pressure on an obstacle is more important for a flow with the same characteristics.
\end{abstract}

\section{Introduction}

Lahars present a mixture of sediments and water originating from volcanoes. The lahar term describes two types of flow: debris flows (DFs) and hyperconcentrated flows (HCFs). DFs are mixture of debris and water with sediment concentration at least 50 vol $\%$ and with a density between 1800 and $2300 \mathrm{~kg} / \mathrm{m}^{3}$. On the other side, HCFs are two phase flows with a sediment concentration between $20 \%$ and $50 \mathrm{vol} \%$ and a density between 1300 and $1800 \mathrm{~kg} / \mathrm{m}^{3}$. Lahars change from HCFs to DFs at solid concentration exceeding $50 \%$ by volume ([1-2]). As a result of global warming, mudslides and flash floods (lahars) are increasingly frequent and expose urbanized areas to a significant risk. For this reason, we are studying the city of Arequipa (Peru) which regularly suffers this type of phenomenon since it is located at $17 \mathrm{~km}$ from the summit of the volcano El Misti . The residential areas exposed to this risk generally include poor populations and structures made of rudimentary masonry. Hence, it is necessary to map the risk through vulnerability quantification.

\section{Objectives}

Our general approach proposes a lahar modelling that better describes the effect of particles. This model is used to simulate the interaction of lahar with typical structures: housing, infrastructures.We can then assess the vulnerability of structure based on the stress level calculated from the flow impact pressure. In this paper, a granular-fluid model is proposed based on DEM and a separate CFD calculation results, to estimate the impact of lahar on a pillar taking into account the coarsest part. The influence of the particle shape is studied by using respectively, for the solid phase: spherical particles called "balls" and polyhedral rigid blocks called "rblocks".

\section{Numerical approach}

Both numerical and experimental ([3-4]) models exist for estimating impact pressure of DF on obstacles. These models are based on different approaches ranging from: (1) purely granular models to simulate a dry granular flow by using DEM [5]; (2) fluid-granular models to simulate the fluid-grain mixture by coupling for example DEM-LBM (Lattice Boltzmann Method) [6]; (3) to purely fluid by using for example Smoothed Particle Hydrodynamics SPH [7]. Simplified ("fluid only" or "granular only") approaches are usually numerically efficient, contrary to coupled approaches which have very high computing times.

The model presented in this paper is an intermediate between purely granular and purely fluid and aims to simulate the impact of lahar at the scale of the structure, locally around it, with a reasonable calculation time [9].

\footnotetext{
* Rime Chehade: rime.chehade@uca.fr
} 


\subsection{Lahar modelling}

The coarsest fraction of the flow, i.e. grains and blocks, is explicitly modelled with DEM by using PFC3D software. Particles are distributed within a given size distribution with a minimal diameter $\mathrm{d}_{\min }$ and a maximal diameter $\mathrm{d}_{\max }$ (Figure 1).

The fluid phase consists ofa mixture of water and fine particles: this phase is modelled with a CFD code and generates two effects on the particles in the DEM model: buoyancy effect and drag force. All other interactions are neglected. Fluid phase plays a major role to move the flow of particles. Telemac $3 D$ is used to simulate the fluid velocity vectors and the free surface height basing on 3D Navier-Stokes equations [9].

Drag force $F_{d}$ represents the effect of the fluid on a particle resulting from the difference of velocity between them. $\mathrm{F}_{\mathrm{d}}$ can be written as follows:

$$
F_{d}=\frac{1}{2} C_{d} \rho_{f} \frac{\pi d^{2}}{4}\left\|V_{f}-V_{b}\right\|\left(V_{f}-V_{b}\right)
$$

where $\rho_{\mathrm{f}}$ is the density of the fluid $\left(\mathrm{kg} / \mathrm{m}^{3}\right), \mathrm{d}$ is the particle diameter $(\mathrm{m}), \quad \mathrm{C}_{\mathrm{d}}$ is the drag coefficient expressed as a function of the particle Reynolds number, $V_{f}$ the velocity vector of the fluid and $V_{b}$ the velocity of the immersed particle.

A correction factor $n^{-\xi+1}$ depending on the solid fraction (n) in the flow is applied to $F_{d}$ in Eq. 1, where $\xi$ is a coefficient ranging between 3.4 and 3.7 [10]. Considering this correction, the new drag force is used as follows:

$$
\mathrm{F}_{\mathrm{d}}=\frac{1}{2} \mathrm{C}_{\mathrm{d}} \rho_{\mathrm{f}} \frac{\pi \mathrm{d}^{2}}{4}\left\|\mathrm{~V}_{\mathrm{f}}-\mathrm{V}_{\mathrm{b}}\right\|\left(\mathrm{V}_{\mathrm{f}}-\mathrm{V}_{\mathrm{b}}\right) n^{-\xi+1}
$$

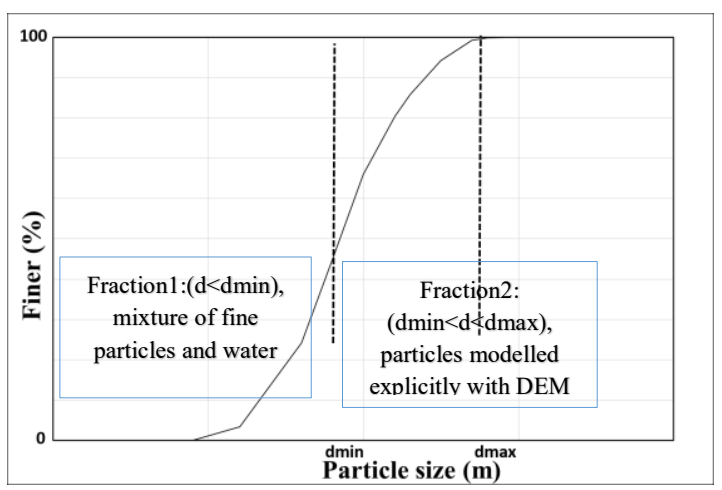

Fig. 1. Lahar granulometry modelling strategy.

\subsection{Model procedure}

A channel is supplied with particles through a chamber containing a representative elementary volume (REV) which is repeated periodically (Figure 2). This REV exhibits the packing characteristics that particles are expected to adopt in the flow with a porosity of 0.5 . Particles in the supply chamber move towards the channel entrance with a fixed velocity equal to the velocity of the fluid phase, in the direction of the channel axis, then are released once they enter the channel. Particles can move in the channel according to the fluid forces applied during the entire flowing process.

The channel is straight with a rectangular cross section with a low gradient slope $(2 \%)$ in the direction of the flow path axis. The channel is $10 \mathrm{~m}$ wide and 25 $\mathrm{m}$ long.

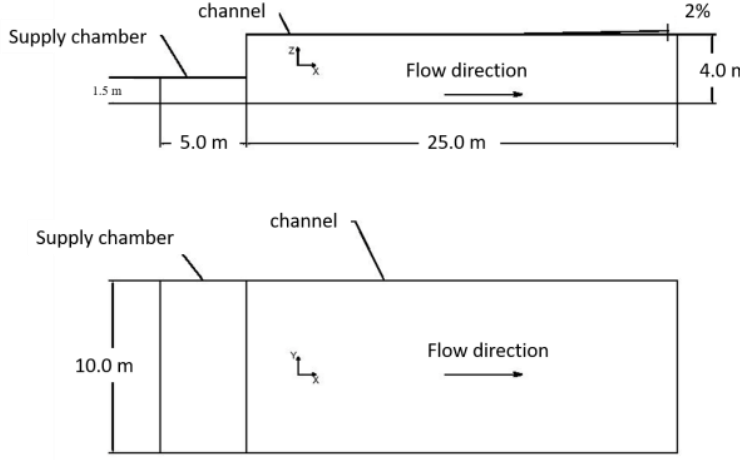

Fig. 2.: Sketch presents the numerical channel.

\subsection{Model parameters}

The parameters of the flow are derived from an example evaluated in Arequipa [8]. The grain-size distribution is uniformly distributed between $\mathrm{d}_{\min }=0.10 \mathrm{~m}$ and $\mathrm{d}_{\max }=$ $0.40 \mathrm{~m}$. We assume $d_{\min }$ the threshold between fine and coarse particles to shorten the computation time [6].

The characteristics of the simulated DF and the strategy used are given in the Figure 3. A parametric study is carried out to analyse the influence of each parameter described below: particles density, fluid density, dynamic viscosity, friction coefficient (particleparticle and particle-wall) and rolling resistance. After performing a parametric study based on 12 simulation cases, the numerical parameters are calibrated. In the end, the flow presents: a solid concentration of $55 \%$, an apparent density of $1867 \mathrm{~kg} / \mathrm{m}^{3}$ and a flow rate of 40 $\mathrm{m}^{3} / \mathrm{s}$. Based on these orders of magnitude, the case of DFs is taken into account in this study.

\begin{tabular}{|c|c|c|c|}
\hline \multicolumn{2}{|c|}{ Property calibration } & \multicolumn{2}{|c|}{$\begin{array}{c}\text { DFs characteristics } \\
\text { obtained }\end{array}$} \\
\hline Density of particles $\left(\mathrm{kg} / \mathrm{m}^{3}\right)$ & 2500 & \multirow{2}{*}{$\begin{array}{c}\text { Solid } \\
\text { concentration } \\
(\%)\end{array}$} & \multirow{2}{*}{55} \\
\hline Fluid density $\left(\mathrm{kg} / \mathrm{m}^{3}\right)$ & 1100 & & \\
\hline Dynamic viscosity (Pa.s) & 0.048 & \multirow{2}{*}{$\begin{array}{c}\text { Apparent } \\
\text { density } \\
\left(\mathbf{k g} / \mathbf{m}^{3}\right)\end{array}$} & \multirow{2}{*}{1867} \\
\hline Friction particle-particle & 0.4 & & \\
\hline Friction particle-wall & 0.0 & \multirow{2}{*}{$\begin{array}{c}\text { Flow rate } \\
\left(\mathrm{m}^{3} / \mathbf{s}\right)\end{array}$} & \multirow{2}{*}{40} \\
\hline Rolling resistance & 0.2 & & \\
\hline
\end{tabular}

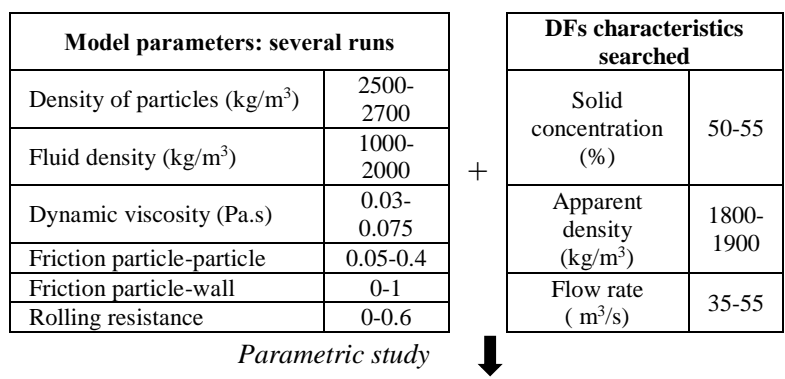

Fig. 3. Characteristics of the simulated DFs.

\section{Impact pressure results}

\subsection{Numerical channel with a pillar}

Once the flow calibrated without obstacle, the DFs model is used to evaluate the total pressure of DFs on a pillar. In this case, the pillar has a square shaped 
horizontal section $(1 \mathrm{~m} \mathrm{x} 1 \mathrm{~m})$ and is located at $15 \mathrm{~m}$ from the entrance of the channel (Figure 4).

\subsection{One way coupling CFD-DEM}

In the case of the channel without obstacle, the fluid velocity field is considered uniform. For the channel with the pillar, we have to compute the velocity fluid vectors and the free surface along the channel. By using $C F D$, we imposed boundary conditions with a constant flow rate at the entrance and a constant flow height at the exit of the channel. We obtained the fluid velocity field and the free surface at each mesh point used in the fluid simulation. Then, these data are used in the $P F C 3 D$ simulation to compute drag forces and then obtain the particle-fluid interactions during the simulation process. CFD calculation is only made once before the DEM calculation.

\subsection{Influence of the shape of the particles}

\subsubsection{Reference case}

The reference case presents a flow density of 1867 $\mathrm{kg} / \mathrm{m}^{3}$, a velocity of $3 \mathrm{~m} / \mathrm{s}$ and a flow height of $1.5 \mathrm{~m}$ (Froude number $=0.78$ ).

The calibration process uses the same procedure with the same particle size distribution for both particles' shapes: balls (Fig.4a) and r-blocks (Fig.4b). For the r-block particles, we used one type of r-blocks with1 80 facets, also representing a rougher material. Rblock particle shape was generated in Rhino program (a CAD program) then imported into $P F C$. Impact results are presented for each case after a simulation duration of $12 \mathrm{~s}$.
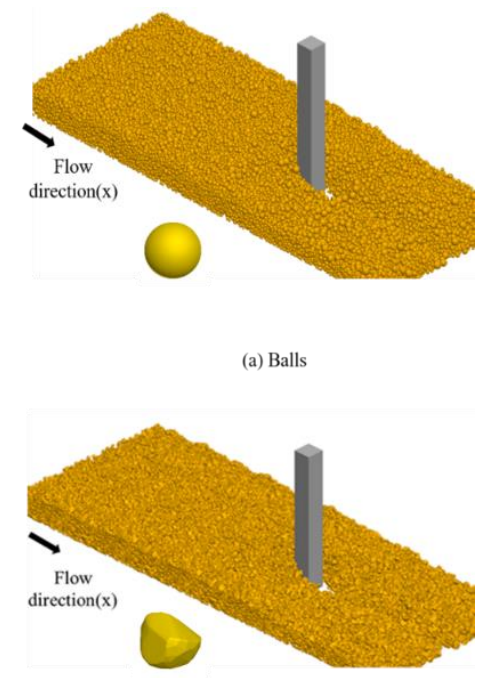

(b) Rblocks

Fig. 4.: Two simulations with different shapes of particles: (a) balls and (b) r-blocks.

The normal forces are represented as the histogram of normal forces exerted on the pillar. Figure 5 shows the diagram of the forces exerted by the spherical particles on the pillar. This diagram presents the distribution of forces according to the intensity classes: The highest population is between 1000 and $10000 \mathrm{~N}$.

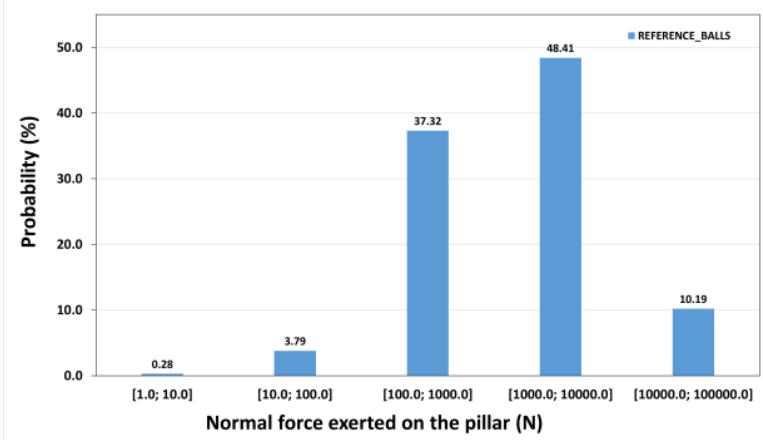

Fig. 5.: Histogram of normal forces for the spherical particles.

The influence of particles shape on the impact pressure is then studied: Figure 6 shows the distribution of the pressure applied by particles and calculated directly in the DEM model from the spatial distribution of the contact forces between the particles and the pillar, as a function of time and for both spherical particles and r-blocks cases. The first particle impacts the pillar after $5 \mathrm{~s}$ in both cases. From $\mathrm{t}=5 \mathrm{~s}$ to $\mathrm{t}=9 \mathrm{~s}$, the stress progressively increased. After $t=9 \mathrm{~s}$, and despite rapid fluctuations, the impact stress curve is stabilized. The total force exerted on the entire pillar is greater for $r$ blocks than for balls.

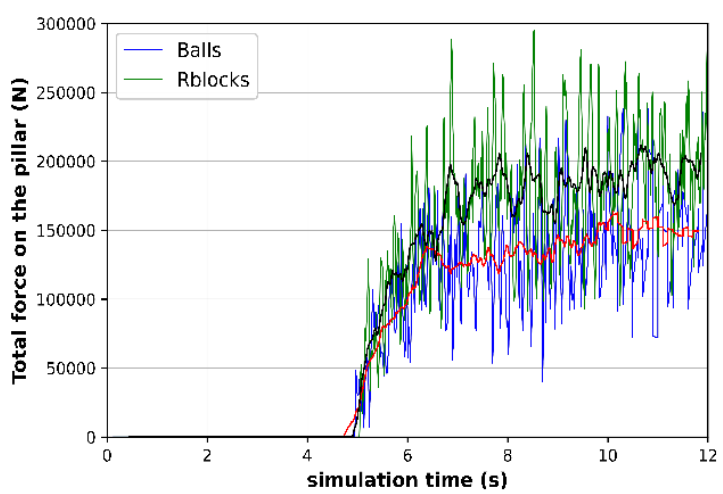

Fig. 6.: Total force applied by particles on a pillar versus simulation time. The black curve shows a moving average window (filter) with $\mathrm{k}=10$ for r-blocks ant the red one for balls.

\subsubsection{Impact pressure}

Pressure diagrams are presented for both cases: spherical particles and r-blocks. Figure 7 shows the evolution of the total pressure (particles added to the static fluid pressure) as a function of the height on the pillar for each case; the standard deviation of the pressure measured from $\mathrm{t}=9 \mathrm{~s}$ to $\mathrm{t}=12 \mathrm{~s}$ is included in the figure. For both cases, the total DFs pressure is stronger at the bottom of the pillar. The maximum pressure exerted on the pillar is higher with the r-blocks than with balls: the pressure on the pillar induced by rblocks is 1.3 times greater than by balls. With a more angular and multi-faceted geometry, the contact area is larger than with a simple and homogeneous shape, hence the impact is higher. Moreover, with spherical particles, the spheres can impact with the pillar and pass 
to the other side more easily than r-blocks: they can slide more easily along the pillar while the r-blocks due to their shape can be slowed down. The r-block displacements are prevented by other r-blocks in contact with them. The time to go around the pillar is more important for an r-block than a sphere, therefore the pressure is higher. This figure also shows that the accumulation height of particles is similar for both cases.

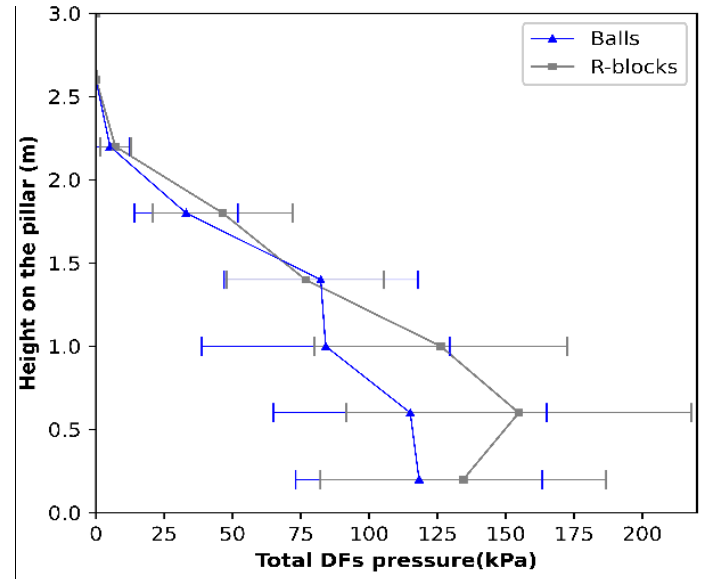

Fig. 7.: Total pressure applied by particles: balls and r-blocks on the pillar including horizontal bars showing time scattering.

\subsubsection{Variation of DFs velocity}

The DFs velocity is varied in the model to estimate its influence on the impact of the DFs on the obstacle. Figure 8 shows the evolution of the total impact pressure induced by balls and r-blocks for three different velocities considering a same flow height of $1.5 \mathrm{~m}$. As expected, we noticed that whatever the shape of the particle, the impact pressure increases with velocity flow [9]. With a higher velocity, the drag force applied on the particles increases as well as the turbulence around the obstacle. About the effect of particle shape, we observe the same result as for the reference case: angular r-blocks induce higher pressures than spherical particles. This figure also confirms that the accumulation height of particles is not influenced by the shape of the particles.

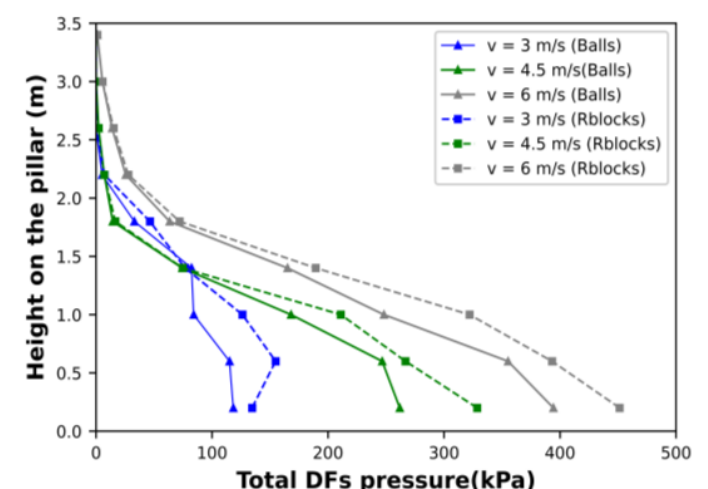

Fig. 8.: Total stress induced by DFs versus the flow height on the pillar for three cases of flow velocity (dashed curve presents the results of r-blocks).

\section{Conclusions}

The numerical model presented is able to reproduce a simplified description of the DFs phenomena. This model presents two phases: particles simulated explicitly by using DEM and the fluid phase which allows to move the particles as the velocity vectors and the free surface are obtain by using CFD. This model can evaluate the total pressure applied by DFs on an obstacle. This model is presented with a specific flow characteristic: density, velocity, solid concentration, and height. However, all these parameters can be easily modified in the simulations. Each parameter related to the flow part (shape of particle, size...) or the structure part (orientation, position...) can influence the impact force results of DFs.

In this paper, the influence of the DF velocity and the particle shape are presented. The particle contact on an obstacle is influenced by the particle shape (spherical/ convex polyhedral shape). The particle shape is a parameter that influences the impact but is not detailed in the literature. The results of simulations show that the impact pressures are higher with an r-block than with a ball. A r-block particle may require more time to get around the obstacle compared to a spherical shape, which modifies the contact and its pressure. Finally, the impact pressure is higher as the DFs velocity is higher which is consistent with existing empirical models (pressure function of velocity).

Each simulation presents a different values of impact pressure that can be used to assess the vulnerability of constructions.

\section{References}

[1] EE. Doyle, SJ. Cronin, JC. Thouret, GSA. Bulletin. 123, 7-8 (2011)

[2] V. Manville, JJ. Major, SA. Fagents Modeling volcanic processes, chapter 14, 300-330 (2013)

[3] L. Bugnion, BW. McArdell, P. Bartelt, C. Wendeler, Landslides 9, 2 (2012)

[4] L. Gao, L. M. Zhang, H. X. Chen, Eng. Geol. 226, 236-244 (2017)

[5] A. Albaba, S. Lambert, F. Nicot, B. Chareyre, Granul. Matter 17, 603-616 (2015)

[6] A. Leonardi, FK. Wittel, M. Mendoza, R. Vetter, HJ. Herrmann, Comput-Aided Civ and Inf. 31, 5 (2016)

[7] Z. Dai, Y. Huang, H. Cheng, Q. Xu, Landslides 14, 3 (2017)

[8] SR. Mead, C. Magill, V. Lemiale, JC. Thouret, M. Prakash, NHESS 17, 5 (2017)

[9] R. Chehade, B. Chevalier, F. Dedecker, P. Breul, JC. Thouret, (to be published)

[10] T. Zhao, GT Houlsby, S. Utili, Granul. Matter 16, 921-932 (2014) 